\title{
A Search for Unrecognized Carbon-Enhanced Metal-Poor Stars
}

\section{Vinicius M. Placco ${ }^{1}$, Catherine R. Kennedy ${ }^{2}$, Silvia Rossi ${ }^{1}$, Timothy C. Beers ${ }^{2}$, Norbert Christlieb ${ }^{3}$ and Thirupathi Sivarani ${ }^{4}$}

${ }^{1}$ Departamento de Astronomia - Instituto de Astronomia, Geofísica e Ciências Atmosféricas, Universidade de São Paulo, São Paulo, SP 05508-900, Brazil email: vmplacco@astro.iag.usp.br

${ }^{2}$ Department of Physics \& Astronomy and JINA: Joint Institute for Nuclear Astrophysics, Michigan State University, East Lansing, MI 48824, USA

${ }^{3}$ Zentrum für Astronomie der Universität Heidelberg, Landessternwarte, Königstuhl 12, 69117, Heidelberg, Germany

${ }^{4}$ Indian Institute of Astrophysics, 2nd block, Koramangala, Bangalore 560034, India

\begin{abstract}
We have developed a new procedure to search for Carbon-Enhanced Metal-Poor (CEMP) stars from the Hamburg/ESO (HES) prism-survey plates. This method uses an extended line index for the $\mathrm{CH}$ G-band, which we demonstrate to have superior behavior when compared to the narrower G-band index formerly employed for these spectra.

A first subsample, biased towards brighter stars $(\mathrm{B}<15.5)$, has been extracted from the scanned HES plates. After visual inspection (to eliminate spectra compromised by plate defects, overlapping spectra, etc., and to carry out rough spectral classifications), a list of 669 previously unidentified candidate CEMP stars was compiled. Follow-up spectroscopy for a pilot sample of 132 candidates was obtained on the SOAR $4.1 \mathrm{~m}$ telescope. Our results show that most of the stars observed lie in the targetted metallicity range, and possess prominent carbon absorption features at $4300 \AA$. The success rate for the identification of new CEMP stars is $\sim 50 \%$ for $[\mathrm{Fe} / \mathrm{H}]<-2.0$. For stars with $[\mathrm{Fe} / \mathrm{H}]<-2.5$, the ratio increases to $100 \%$.
\end{abstract}

Keywords. stars: abundances, stars: carbon, stars: Population II, Galaxy: halo, surveys

\section{Introduction}

Although CEMP stars have been found previously among the candidate metal-poor stars selected from the HES, the selection on metallicity undersamples the population of intermediate-metallicity CEMP stars $(-2.5 \leqslant[\mathrm{Fe} / \mathrm{H}] \leqslant-1.0)$; such stars are of importance for constraining the onset of the s-process in metal-deficient Asymptotic Giant-Branch stars (thought to be associated with the origin of carbon for roughly $80 \%$ of CEMP stars). The new candidates also include substantial numbers of warmer carbon-enhanced stars, which were missed in previous HES searches for carbon stars due to selection criteria that emphasized stars with cooler temperatures.

The primary goal of the present work is to demonstrate the efficacy of searching for these intermediate-metallicity CEMP stars. The inclusion of warmer carbon-enhanced candidates (which do not exhibit $\mathrm{CN}$ and $\mathrm{C}_{2}$ bands) also enables investigations between the observed levels of carbon enhancement and evolutionary stage. It should also be kept in mind that the inventory of ultra $([\mathrm{Fe} / \mathrm{H}]<-4.0)$ and hyper $([\mathrm{Fe} / \mathrm{H}]<-5.0)$ metalpoor stars is almost certainly incomplete. Such extreme stars may have been overlooked in previous searches due to noisy spectra in the region of CaII $\mathrm{K}$ on objective-prism plates, but they could reveal themselves by the presence of strong $\mathrm{CH}$ G-bands that are commonly associated with the most metal-deficient stars. 
We have developed a new line index for the region of the carbon G-band at $4304 \AA$,

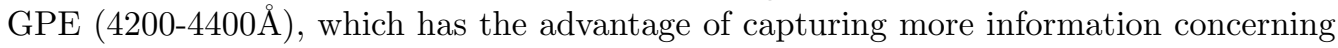
the abundance of carbon, since its width takes into account the wings of the band, which includes other nearby carbon features, and is not subject to confounding of previously employed narrower indices, due to sidebands that fall in regions of the spectrum for which carbon features are present.

\section{Metallicity and carbon abundances}

Validation of our selected CEMP candidates is an important part of this pilot study. For this purpose, we have obtained medium-resolution $(R \sim 1500)$ optical spectra for 132 of our 669 CEMP candidates with the new Goodman high-throughput spectrograph on the SOAR 4.1m telescope. After gathering and reducing the data, we also obtained firstpass estimates of the the atmospheric parameters using the SEGUE Stellar Parameter Pipeline (SSPP - Lee et al. 2008) and [C/Fe].

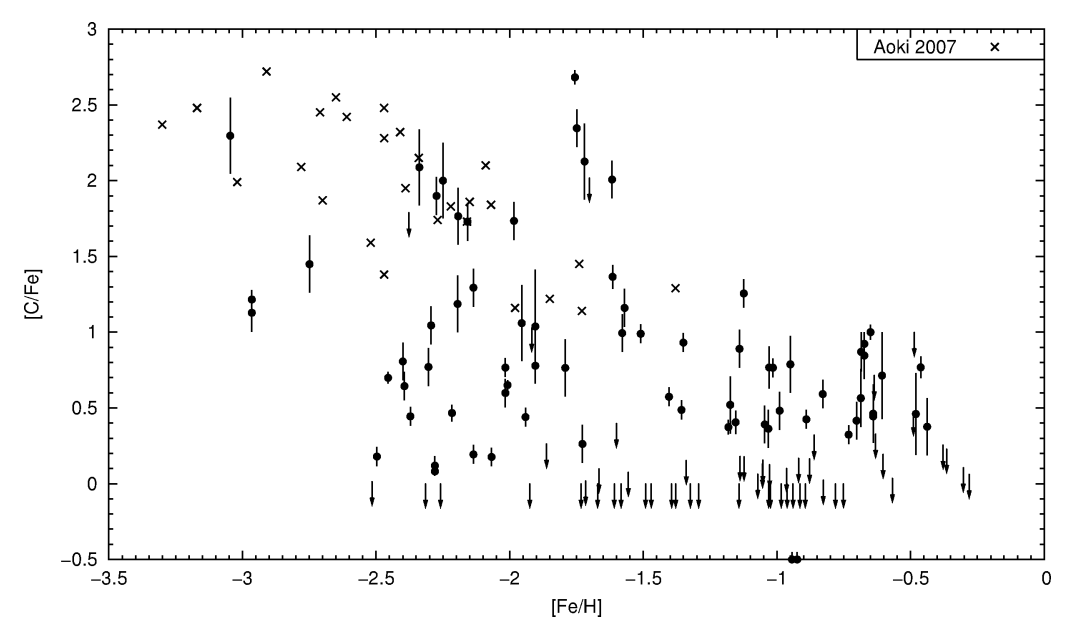

Figure 1. Behavior of the metallicity with the carbon abundance $[\mathrm{C} / \mathrm{Fe}]$ for the observed candidates and the stars from Aoki et al. (2007). The arrows represent upper limits.

Since the goal of this work is to target CEMP stars, we have confirmed that the high fraction found, based on our selection method, is more than two times higher than the one claimed in Lucatello et al. (2006) for all stars with $[\mathrm{Fe} / \mathrm{H}]<-2.0$ observed to date $(20 \%)$. It is also important to note that the majority of metal-poor stars in our candidate pool with $[\mathrm{Fe} / \mathrm{H}]<-1.0(\sim 70 \%)$ present carbon enhancements $([\mathrm{C} / \mathrm{Fe}]>+0.5)$.

\section{Acknowledgements}

V.M.P. and S.R. acknowledge CAPES (PROEX), CNPq, JINA and FAPESP funding (2007/04356-3). C.R.K. and T.C.B. acknowledge JINA.

\section{References}

Aoki, W., Beers, T. C., Christlieb, N. et al. 2007, ApJ, 655, 492

Christlieb, N., Schörck, T., Frebel, A. et al. 2008, A\&A, 484, 721

Beers, T. C. \& Christlieb, N. 2005, ARA\&A, 43, 531

Lee, Y. S., et al. 2008, AJ, 136, 205

Lucatello, S., Beers, T. C., Christlieb, N. et al. 2006, ApJ, 652, L37 